\title{
Prologue and Invitation to Participate in a Forum on the Frequency Dependence of Seismic Q
}

\section{Brian Mitchell ${ }^{1}$}

Few aspects of seismic wave propagation have aroused as much disagreement among seismologists as that of seismic wave attenuation (or its inverse Q). Areas of disagreement include (1) the physical causes of the attenuation, (2) the relative contributions of scattering and intrinsic absorption at various frequencies during propagation, and (3) the nature of the frequency dependence of Q.

Seismologists, in their publications, have largely avoided addressing these disagreements by ignoring all papers promoting a perspective differing from their own. This forum seeks to remedy that situation for issue (3), the nature of $\mathrm{Q}$ frequency dependence.

Igor Morozov has recently published a number of papers in which he concludes that there is no compelling reason to think that $\mathrm{Q}$ varies at all with frequency. This revolutionary result, if correct, negates almost all of the work on $\mathrm{Q}$ frequency dependence that seismologists have reported going back to at least the mid-1970s.

In the spirit of having an open and full discussion of the nature of Q frequency dependence (or the lack thereof) I have convened a forum to discuss $\mathrm{Dr}$ Morozov's recently proposed methodology. He has agreed to defend his method and ensuing results and Dr Jiakang Xie has agreed to argue the case against the new approach and to defend the traditional way of looking at $\mathrm{Q}$ frequency dependence.

If you would like to participate in this forum I invite you to send your comments to me (bmitche8@slu.edu) in an email attachment. Comments will appear in a special section on Forum Responses in the November issue of PAGEOPH. Please restrict all comments to one manuscript page or, at most, two.

\footnotetext{
${ }^{1}$ Department of Earth and Atmospheric Sciences, Saint Louis University, 3642 Lindell Blvd, Saint Louis, MO 63122, USA. E-mail: bmitche8@slu.edu
} 